\title{
CONTEMPORARY ASPECTS OF THE EPIDEMIOLOGICAL SURVEILLANCE OF SOCIALLY SIGNIFICANT TICK-BORNE INFECTIONS (TBIS) IN VARNA REGION
}

\author{
Rumen Konstantinov ${ }^{1}$, Tsonko Paunov ${ }^{1}$, Svetla Staneva ${ }^{1}$, Anka Baeva ${ }^{2}$ \\ ${ }^{1}$ Department of Hygiene and Epidemiology, Faculty of Public, Medical University of Varna \\ ${ }^{2}$ RHI-Varna
}

\begin{abstract}
The modern system for epidemiological surveillance of tick-borne infections (TBIs) in the Varna region requires a systematic analysis of the epidemic process.

AIM: The aim of this article is to trace the evolution in parameters of the parasitic system in Lyme Borreliosis, Boutonneuse fever, Crimean-Congo hemorrhagic fever, and Q-fever in the Varna region for the period 20012017 and the activities of the epidemiological surveillance system.

MATERIALS AND METHODS: Data from active epidemiological studies and analyses of TBIs in the region of Varna and Bulgaria for the period 2001-2017 were used; as well as epidemiological and entomological methods, statistical and graphic analyses, nosogeographic analyses and others.

RESULTS: Data from the epidemiological analysis determined the continuing health and social significance of registered TBIs in the Varna region during the considered period. High levels of morbidity in Lyme disease (LD) and Boutonneuse fever (BF) with characteristic summer seasonality, correlated with the activity of vectors - reservoirs (Ixodes ticks), have been established. The system of epidemiological surveillance includes events for effective dispensary monitoring of the bitten by a tick; collecting complete epidemiological information about the area where the bite has occurred; preparation of epidemiological analysis of the morbidity of transmissible infections on the territory of the Varna region with emphasis on Lyme borreliosis, Boutonneuse fever, Q fever, Crimean-Congo hemorrhagic fever (CCHF), surveillance over the species composition and territorial distribution of tick populations and expanding the tick control activities in the region. There is a timely diagnosis and treatment of the registered patients. It is necessary to expand and deepen the system of epidemiological surveillance with the proposed strategy for surveillance and control of socially significant TBIs in the Black Sea region of the Republic of Bulgaria.
\end{abstract}

Keywords: tick-borne infections, Varna region, epidemiological surveillance

\author{
Address for correspondence: \\ Rumen Konstantinov \\ Faculty of Public Health \\ Medical University of Varna \\ 55 Marin Drinov St \\ 9002 Varna \\ e-mail:konstant@mail.bg
}

Received: May 28, 2018

Accepted: June 14, 2018

\section{INTRODUCTION}

The epidemiological and socio-economic importance of infectious diseases determines the need of continuous monitoring of the rapidly changing epidemiological situation (surveillance) in order to timely develop measures for the liquidation or to limit the spread of infectious diseases to a certain minimum level (control) (1-4). Epidemiological surveillance and control of socio-significant zoonotic 
tick-borne infections (TBIs) result from the changing environmental, landscape and anthropogenic conditions that shape the epidemiological features of the TBI and the need for an adequate response from the healthcare system (5-9). The present work is a natural continuation of the systemic epidemiological observations and analyses of the socially significant TBIs in the Varna region, on the basis of which a Preventive Strategy for Supervision and Control of TBIs is being implemented and synchronized with the $\mathrm{Na}-$ tional Program for Prevention and Control of Vector Transmitted Infections in Humans in Bulgaria for 2014-2018 (3,7,8). Based on the epidemiological surveillance data, decision makers have the opportunity to clarify the encountered problems, to find the exposed humans and to determine the orientation of program activities and preventive measures. A certain set of epidemiological data is required to solve the priority problems for the protection of the health of the population and the mechanism of epidemiological surveillance itself is extremely important for the evaluation of the effectiveness of the preventive work $(1,3,5-8,10)$.

\section{AIM}

The aim of this article is to track the evolution of parameters of the parasitic system in Lyme borreliosis (LB), Boutonneuse fever (BF), Crimean-Congo haemorrhagic fever (CCHF) and Q-fever (QF) in Varna region (VR) for the period 2001-2017 and the activities of the epidemiological surveillance system based on the National Program for Prevention and Control of Vector Transmitted Infections in Humans in the Republic of Bulgaria for 2014-2018 (3).

\section{MATERIALS AND METHODS}

Epidemiologically investigated and registered were 2665 cases of TBI in the Varna region for the period 2001-2017, of which 1672 cases of BF, 1006 cases of LB, 34 cases of QF and 4 cases of CCHT. Species-differentiated ticks were removed from bitten people for the period 2002-2017 in VR. The data used was from official statistical digests and documents, as well as from applied epidemiological surveillance of TBI in VR. Epidemiological and entomological methods, statistical and graphical analyses were used.

\section{RESULTS AND DISCUSSION}

The results of studies from a number of authors have found that climate change related to the global warming process has an impact on the epidemiology of vector-borne infectious diseases $(3,7,8,11-17)$. The ecological changes lead to an increase in the reservoirs of infection among the animals and the density of the population of the vectors carriers. Anthropogenic environmental change, the creation of artificial water basins, urbanization and related changes in the urban and suburban environment lead to the creation of permanent parasitic systems and high epidemic risk for humans. $(2,5,7,8,11)$

The main information parameters, representing the evolution of the parasitic system in the socially significant TBIs in VR, track and record the multitude of data forming the parasitic system. The main epidemiological indicators for the period 2001-2017, defining the evolution in the epidemiological significance of $\mathrm{LB}, \mathrm{BF}, \mathrm{CCHF}$ and $\mathrm{QF}$ in VR, are data on vector activity and exposure. It is established that the incidence of transmissible infections in VR for the period 2001-2017 has a downward trend (2001 - 87.72 \%ooo; 2012 -18.73 \%оoo) (Fig. 1).

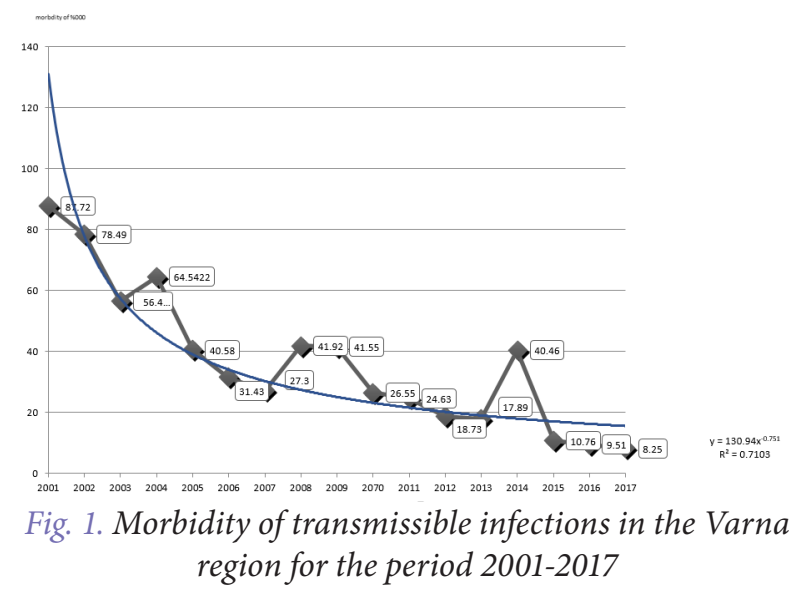

$\mathrm{BF}$ has a leading role in the spectrum of vectorborne infections in VR with $65 \%$ followed by LF 34\% (Fig. 2).

LB morbidity has values up to twice the average for the country, with an equal even course of the formed trend (Fig. 3).

BF morbidity in 2001 was 4 times higher than the country's average, while in 2017 it is almost the 


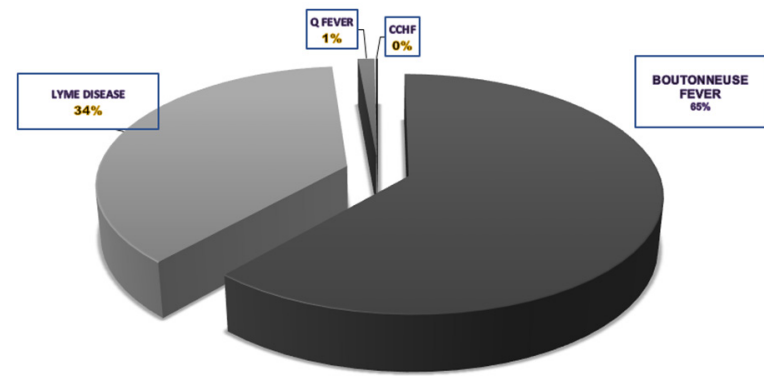

Fig. 2. Structure of registered transmissible infections in Varna region for the period 2001-2017

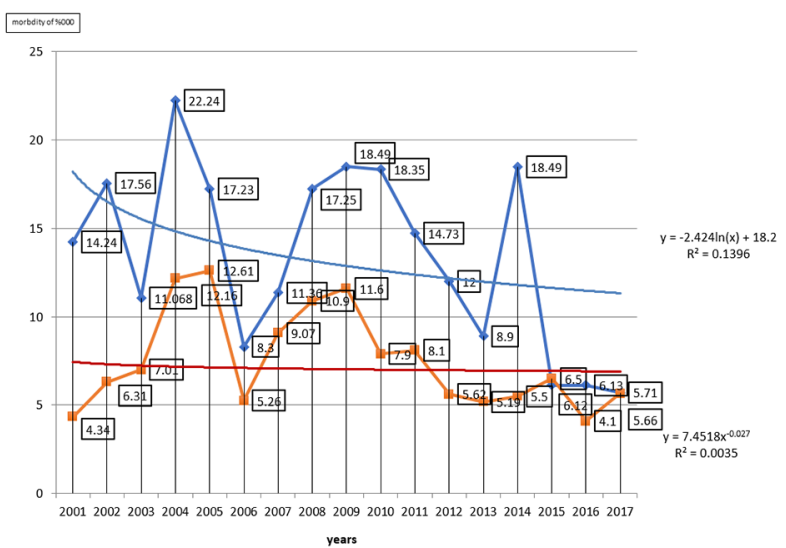

Fig. 3. Morbidity of Lyme disease in the Varna region and Bulgaria for the period 2001-2017

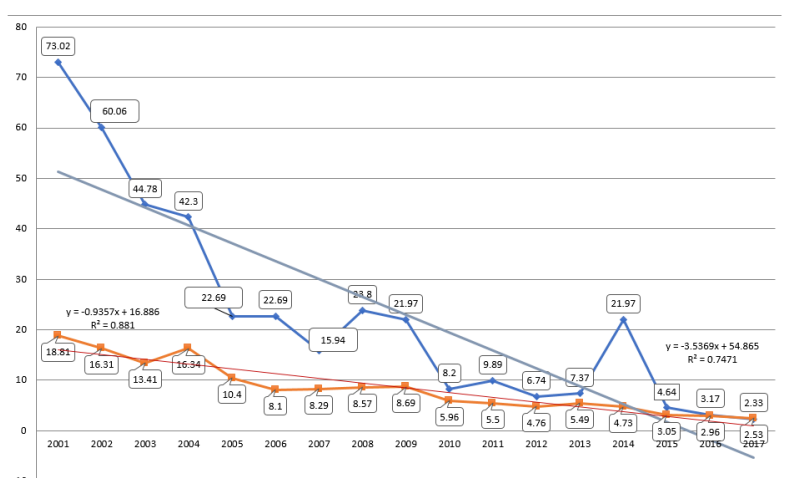

Fig. 4. Morbidity from Boutonneuse fever for the period 2001-2017 in Varna region

same as that of Bulgaria - $6.74 \%$ oooo. A strong regression trend is forming (Fig. 4).

The seasonality of LB in VR is spring-summer with peaks in May, June, and July (Fig. 5) coinciding with the activity season of the vector - I. ricinus ticks. The seasonal distribution of BF in VR is spring-summer with a peak in August, when the highest activity of Rh. sanguineus is reported (Fig. 6).
There is a parallel between the dynamics of the tick populations, the tick-bitten people and the in-

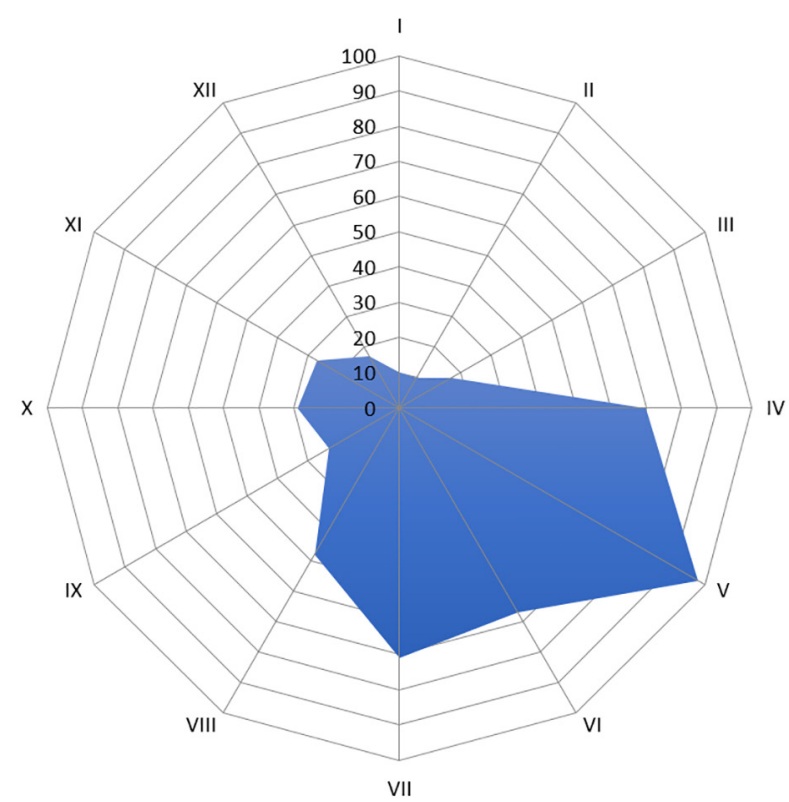

Fig. 5. Seasonality of cases of Lyme disease in the Varna region for the period 2001-2017

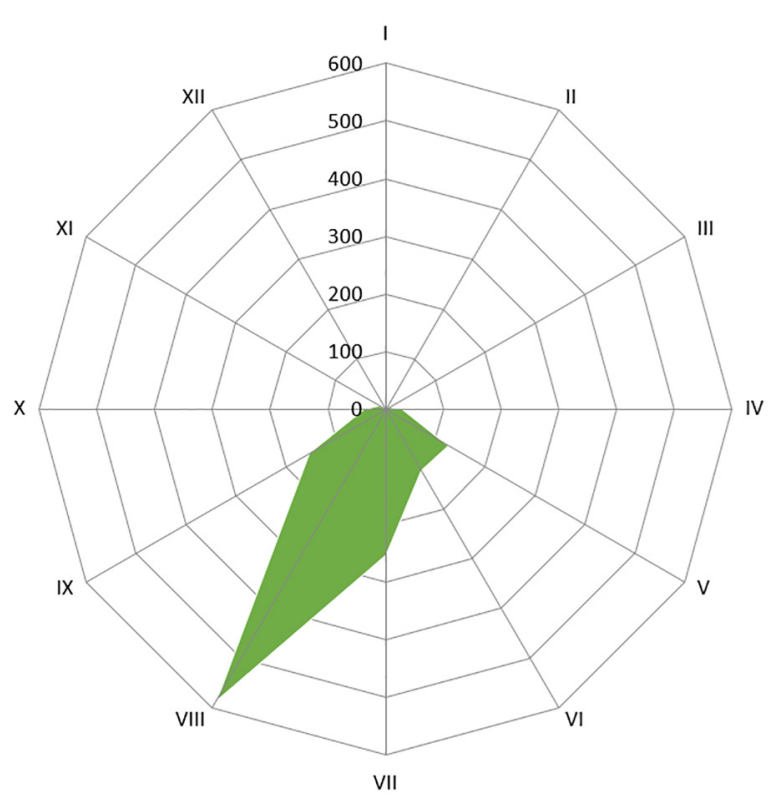

Fig. 6. Seasonality of cases of Boutonneuse fever in the Varna region for the period 2001-2017

cidence of $\mathrm{LB}$ and BF. This relationship is the result of the valent activity of the natural focuses of blood transmissible infections $(7,8,11)$. 
Rumen Konstantinov, Tsonko Paunov, Svetla Staneva et al.

The incidence of CCHF in VR continues to be incidental with single cases without epidemiological regularity and presents data on a possible emerging infection for the region (11,18) (Fig. 7).

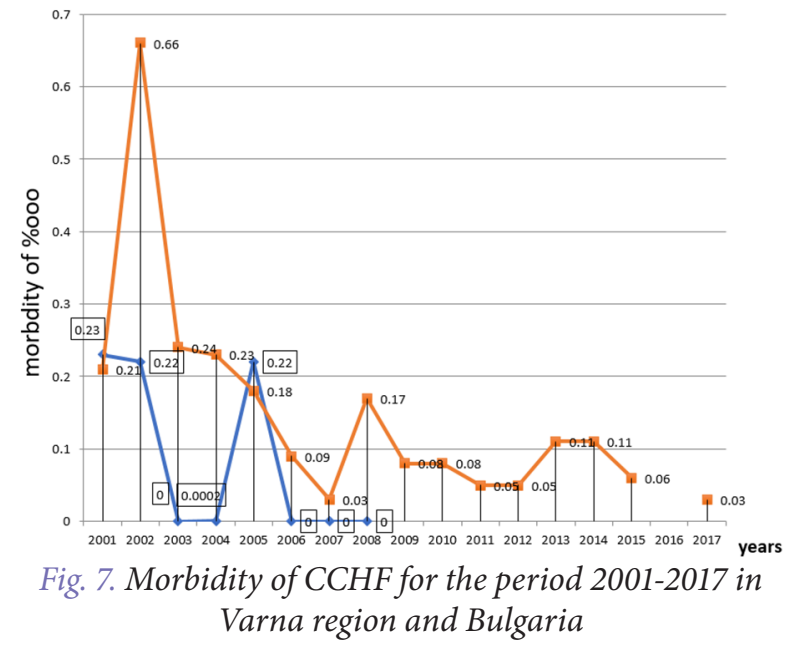

QF has a low degree of diagnostic information and a significant potential for epidemiological research as a phase of epidemiological surveillance. The most common epidemiological studies are retrospective ones of a serologically proven disease in patients with protracted or atypical pneumonia $(10,11)$ (Fig. 8).

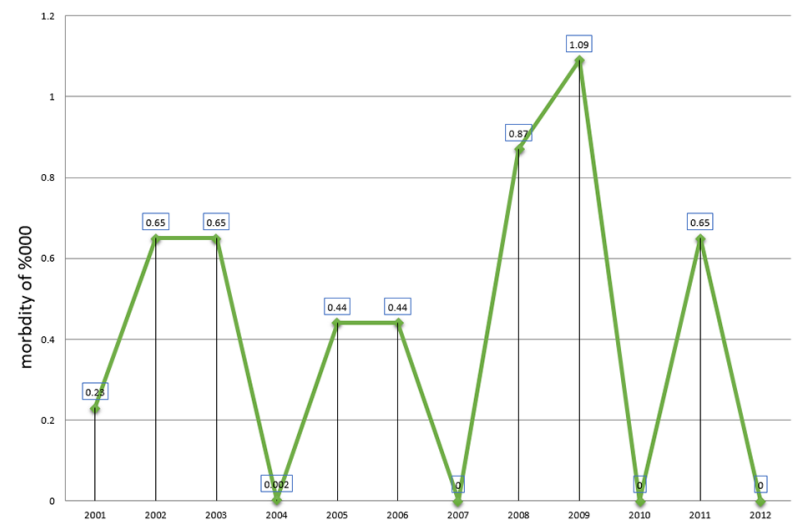

Fig. 8. Morbidity of Q fever for the period 2001 - 2012 in Varna region and Bulgaria

Vector data from the epidemiological surveillance of TBIs in VR:

$\diamond$ The leading tick species for VR are I. ricinus species - 78\%, Rh. sanguineus - 12\%, D. margin- atus $-6 \%, H$. marginatum - 2\%, Rh. bursa - $2 \%$ and others.

$\diamond$ The marked importance of I. ricinus also stands out in the even course of the populations throughout the period $2002-2012$.

$\diamond$ The dynamics of annual variations in the abundance of tick species is determined by the combination of a complex of temperature-humidity constellations (THCs) with a priority of the temperature factor and the humidity conditions $(7,8)$. High average annual temperatures combined with high values of measured precipitations that stimulate tick populations should be taken into account. THCs of low average annual temperatures and low rainfall are both retentive. Among the annual THCs determining are the mild and humid winters, the cool and precipitous summers that stimulate the development of the tick populations and the inverse effect of the cold long winters, hot and dry summers $(7,8)$.

$\diamond$ Seasonal changes in tick populations are dependent on warm and wet spring and summer months and are negatively affected by high summer temperatures combined with low rainfall $(7,8,11)$.

The main land areas with increased number of tick populations (high vector risk) are differentiated, based on favorable ecological conditions for tick life and frequency of tick bites. Their relative borders have been established on the basis of relatively identical living conditions for ticks - extensive construction and ill-kept spaces between the buildings, areas near the beach with increased humidity and abundant vegetation, areas of relatively higher altitude, industrial zones, nature parks and wetlands, forests and shrubs, especially places associated with grazing domestic animals.

Areas with high vector risk are as follows:

$\diamond$ Vladislavovo and Vazrazhdane quarters with large spaces between the buildings;

$\diamond$ Resort areas: Golden Sands, Aladzha Monastery and Eco Park, St. St. Konstantin and Elena;

$\diamond$ Vinitsa and Chaika quarters, and the Sea Garden;

$\diamond$ The settlements around the Galata district Borovets, Chernomorets and Fichoza. The de- 
ciduous forests around Aksakovska Panorama, Sherba and Kamchia Resort;

$\diamond$ Details of bitten from other regions of the country are mostly from Gabrovo and the Elena Balkan region.

The main locations of tick attacks are usually picnic and resort areas, parks and gardens. The results obtained are part of our hypothesis for the emergence of TBIs in the Black Sea region of the Republic of Bulgaria $(7,8)$, based on the following main factors:

1. Nature and climate changes - increased humidity, elevated temperatures, providing favorable conditions for the reproduction of tick populations.

2. Favorable species composition and abundance of tick populations.

3. The presence of many hosts - wild and domestic animals.

4. Existence of favorable socioeconomic conditions:

$\diamond$ Increased population in the coastal zone due to migration and construction of new villages of resort type, tourism and recreation.

$\diamond$ The increased intensity of contacts with carriers, economic and recreational conditions

$\diamond$ Multiple contacts due to agricultural activity, home breeding of animals, dogs, etc. in bad conditions.

In conclusion, it is possible to highlight the complexity of the processes of new emergence of LB in the Black Sea region of the Republic of Bulgaria, where all the factors of the emergence from natural and anthropogenic nature are presented. There is a complex system of temperature and humidity conditions affecting the parasitic system consisting of causative agents and arthropod carriers, animal reservoirs and the epidemiological outcome - the TBI morbidity. These factors should be taken into account when developing a concept for the management of emerging TBI processes $(7,8)$.

Data from epidemiological surveillance represent the continuing health and social significance of registered tick-borne infections in VR during the period under review.
The goals set in the National Program for Prevention and Control of Vector Transmitted Infections in Humans in the Republic of Bulgaria for 20142018 include: reducing morbidity and mortality from vector-transmitted infections and reducing them to single cases in the country; a decrease in mortality and lethality in the country; achieving integrated epidemiological, veterinary and biological surveillance of vector-transmitted infections; conducting targeted prophylactic, anti-epidemic and anti-epizootic measures to reduce the number of vectors in the settlements; establishing a long-lasting trend of reduced morbidity and mortality and progressively reducing the number of people bitten by vectors (ticks and mosquitoes) (3).

In pursuance of the set objectives for the epidemiological surveillance and the control over TBIs in VR, we present the Preventive Strategy and Directions for Control and Prevention of TBIs in VR $(7,8)$. They include a wide range of preventive, research, educational, and application activities on the surveillance and control of TBIs in the region. Their implementation provides an opportunity for epidemiological control over TBIs in VR.

\section{CONCLUSION}

1. Ecological changes, anthropogenic environmental change, urbanization, and other factors lead to the creation of persistent parasitic systems by TBI and high epidemic risk to humans

2. The main epidemiological indicators for the period 2001-2017, defining the evolution of the epidemiological significance of $\mathrm{LB}$ and $\mathrm{BF}$, show high activity of the epizootic and epidemic process, data on vector activity and exposure.

3. CCHF presents data on a possible emerging infection for the region, while QF has a low level of diagnostic information and a significant potential for epidemiological research.

4. The dynamics of annual variations in the abundance of tick species is determined by the combination of a complex of THCs with a priority of the temperature factor and the humidity conditions.

5. Data from the epidemiological surveillance represent the continuing health and social sig- 
Rumen Konstantinov, Tsonko Paunov, Svetla Staneva et al.

nificance of registered tick-borne infections in VR during the period under review.

6. The epidemiological control over TBI in VR includes a wide range of preventive, research, educational and applied activities.

\section{REFERENCES}

1. Kozhuharova M. European Union policy to improve the surveillance, control and prevention of communicable diseases. In: Theoretical analysis of modern epidemiology. Sofia: Med. And Fizk; 2006. p. 206-14. (in Bulgarian)

2. Monev V, Parmakova K. Problems of epidemiological surveillance of tick-borne infections in Bulgaria. Infectiology. 2008; 55(2):43-6. (in Bulgarian).

3. The National Program for prevention and control of vector-transmitted transmissible infections in the people of Bulgaria for 2014-2018. (in Bulgarian).

4. CDC's Vision for Public Health Surveillance in the 21st Century. MMWR. 2012;61.

5. Dimitrova T. Epidemiology and ecology of the natural focal diseases in Bulgaria (1970-2008). Habilitation work. Sofia; 2009. p. 457. (in Bulgarian).

6. Doiceva V, Mitova J, Petrova E, Angelova S, Mincheva Ts. Epidemiological characteristics of some natural focal zoonotic infections in Bulgaria for the period 2000 - 2013. General Medicine. 2014;16(3). (in Bulgarian).

7. Konstantinov R. New and emerging zoonotic tickborne infections in the Black Sea region of Bulgaria (epidemiological, environmental, socio-economic studies). Diss Doc. Varna; 2010. (in Bulgarian).

8. Konstantinov R. Theoretical and applied epidemiology of the emerging zoonotic infections. Dimant; 2011. p. 252. (in Bulgarian).

9. Huss A, Braun-Fahrländer C. Tick-borne diseases in Switzerland and climate change. 2007. Available at: http://www.bvsde.paho.org/bvsacd/cd68/ticksclimate.pdf

10. 1National Programme for the Prevention, control and eradication of animal diseases and zoonoses 2016-2018. (in Bulgarian).

11. Kunchev AI. Studies on the epidemiology of the most common, tick-borne infections in Bulgaria. Auto Diss. Doc. Sofia; 2018. (in Bulgarian).

12. Morens DM, Folkers GK, Fauci AS. The challenge of emerging and re-emerging infectious diseas- es. 2004. Nature. 430(6996):242-9. doi: 10.1038/ nature02759.

13. GEO Year Book 2004/5: An Overview of Our Changing Environment. Available at: http://hdl. handle.net/20.500.11822/8724.

14. Githeko AK, Lindsay SW, Confalonieri UW, Patz JA. Climate change and vector-borne diseases: a regional analysis. Bull World Health Organ. 2000; 78(9): 1136-47.

15. Gubler DJ. Resurgent vector-borne diseases as a global health problem. Emerg Infect Dis. 1998;4(3):442-50. doi: 10.3201/eid0403.980326.

16. Patz JA, Epstein PR, Burke TA, Balbus JM. Global climate change and emerging infectious diseases. JAMA. 1996;275(3):217-23.

17. Sutherst RW. Global Change and Human Vulnerability to Vector-Borne Diseases. Clin Microbiol Rev. 2004;17(1):136-73.

18. Petrova EI. Study on epidemiological and environmental features, prevention and control of Crimean-Congo hemorrhagic fever in Bulgaria. Autodiss. Doc. Sofia; 2015. (in Bulgarian). 